\title{
CONTROLE DE PLANTAS DANINHAS NA CULTURA DO GIRASSOL COM ACLONIFEN APLICADO ISOLADO OU EM MISTURA
}

\author{
Cleber Daniel de G. Maciel $^{1}$, Jamil Constantin² e Rubem Silvério de Oliveira Junior ${ }^{2}$
}

\author{
${ }^{1}$ Pós-graduando. DPV/FCA/UNESP. Fazenda Lageado. Caixa Postal 237. Botucatu, SP 18603-970 maciel@fca.unesp.br \\ ${ }^{2}$ Dr., Professor Depto de Agronomia/UEM. Av. Colombo, 5790. Maringá, PR 87020-900
}

\section{RESUMO}

O trabalho teve como objetivo avaliar a seletividade e a eficácia do aclonifen, isolado ou em mistura com outros herbicidas, no controle em pós-emergência de plantas daninhas na cultura do girassol. O experimento foi conduzido na Fazenda Experimental da Universidade Estadual de Maringá, PR, na safra 1998/99, utilizando o híbrido Morgan 742. O delineamento experimental utilizado foi blocos ao acaso, com nove tratamentos e quatro repetições. Os tratamentos avaliados foram: testemunha sem capina, testemunha capinada, aclonifen ( 780 e $900 \mathrm{~g} / \mathrm{ha})$, quizalofop-p-ethyl ( $75 \mathrm{~g} / \mathrm{ha})$, aclonifen + quizalofop-p-ethyl $(900+75$ e $900+100 \mathrm{~g} / \mathrm{ha})$, aclonifen + fomesafen $(900+25 \mathrm{~g} / \mathrm{ha})$ e aclonifen + chlorimuron-ethyl $(900+2,5 \mathrm{~g} / \mathrm{ha})$. Os tratamentos químicos foram aplicados quando o girassol encontrava-se no estádio V6 a V8, utilizando pulverizador costal pressurizado à base de $\mathrm{CO}$, que proporcionou um consumo de calda de $200 \mathrm{~L} / \mathrm{ha}$. As misturas de aclonifen + quizalofop-p-ethyl e o quizalofopp-ethyl isolâdo foram seletivos ao girassol e excelentes no controle de Cenchrus echinatus, não diferindo significativamente da testemunha capinada. O aclonifen isolado ou em mistura com fomesafen, chlorimuron-ethyl e quizalofop-p-ethyl foi excelente no controle de Commelina benghalensis e Portulaca oleracea, não havendo diferenças entre estes tratamentos e a testemunha capinada. O aclonifen isolado ou em mistura com fomesafen e chlorimuron-ethyl não causou injúrias visuais ao girassol. A convivência das plantas daninhas, principalmente de C. echinatus, dificultou a colheita e reduziu a produtividade do girassol em $50,0 \%$.

Palavras-chave: Helianthus annuus, interferência, herbicida, seletividade

\section{ABSTRACT \\ Weed control in sunflower crop with application of aclonifen isolated or in mixture}

The aim of this work was to evaluate the selectivity and the efficacy of aclonifen, isolated or in mixture with other herbicides, in postemergence weed control in a sunflower crop. The experiment was conducted in 1998/99, at the Experimental Farm of Maringá State University, Paraná State, using the hybrid Morgan 742. The experimental design was randomized block with nine treatments and four replications. The evaluated treatments were: control (with and without hoeing), aclonifen (780 and $900 \mathrm{~g} / \mathrm{ha})$, quizalofop-p-ethy (75 g/ha), aclonifen + quizalofop-p-ethy $(900+75$ and $900+100 \mathrm{~g} / \mathrm{ha})$, aclonifen + fomesafen (900 $+25 \mathrm{~g} / \mathrm{ha})$ and aclonifen + chlorimuron-ethyl $(900+2.5 \mathrm{~g} / \mathrm{ha})$. The treatments were applied when the sunflower plants were in V6 to V8, using a carbon dioxide-pressurized backpack sprayer, with a spray volume of $200 \mathrm{~L} / \mathrm{ha}$. The mixtures of aclonifen + quizalofop-p-ethyl and the quizalofop-p-ethyl alone were seletive for sunflower and efficient in Cenchrus echinatus control, not differing significantly for the control. Aclonifen alone or in mixture with fomesafen, chlorimuron-ethyl and quizalofop-pethyl were efficient in the control of Commelina benghalensis and Portulaca oleracea not showing differences between these treatments and the control. Aclonifen alone or in mixture with fomesafen and chlorimuron-ethyl did not cause visual injury to the sunflower crop. The presence of weeds, especially C. echinatus, hindered the harvest and decreased the sunflower yield up to $50.0 \%$.

Key words: Helianthus annuus, interference, herbicide, selectivity. 


\section{INTRODUÇÃO}

O girassol (Helianthus annuus L.) é uma oleaginosa de características agronômicas importantes, com maior resistência a seca, ao frio e ao calor que a maioria das espécies cultivadas e, por apresentar ampla adaptabilidade às diferentes condições edafoclimáticas, vem sendo utilizada como uma opção nos sistemas de rotação e sucessão de culturas nas regiões produtoras de grãos (Castro et al., 1996).

Dentre as fontes energéticas renováveis, a exploração racional da cultura do girassol representa uma alternativa de grande importância, quer como fonte de proteína de elevado valor biológico para a alimentação humana e de animais, quer pelo excepcional óleo comestível ou combustível extraído dos seus grãos. (Pires, 1994; Balla et al., 1995). A produtividade da cultura do girassol apresenta-se em torno de $1250 \mathrm{~kg} / \mathrm{ha}$, sendo a Iugoslávia o país que apresenta a maior produtividade, $2000 \mathrm{~kg} / \mathrm{ha}$ (Constantin 1994). No Brasil, apesar de existirem híbridos que proporcionam até $2500 \mathrm{~kg} / \mathrm{ha}$, isto não ocorre na maioria das vezes, estando a produtividade média nacional em torno de $600 \mathrm{~kg} / \mathrm{ha}$. Desta forma, o girassol pode ocupar lugar de relevância na agricultura nacional, desde que se desenvolvam artifícios que minimizem a problemática da baixa produtividade da cultura.

De forma semelhante ao que acontece com outras culturas, as plantas daninhas competem com o girassol por água, luz e nutrientes, afetando a produtividade. Os danos que as plantas daninhas causam ao girassol podem variar de 20 a $50 \%$ de perdas na produção de grãos, caso não se faça o controle (Robinson, 1966; Chubb, 1975; Montes de Oca \& Pearsom, 1987; Trasmonte \& Duarte, 1991; Constantin, 1994). A época de controle também é um fator muito importante. $\mathrm{O}$ período em que a cultura do girassol deve permanecer livre da convivência com as plantas daninhas varia entre a quarta e sexta semana após a semeadura. Castro et al. (1996) e Ungaro (1978) recomendam que a cultura deve permanecer no limpo até cerca de 30 e 40 dias após a semeadura, respectivamente.

Ainda é difícil o controle químico de plantas daninhas na cultura do girassol no Brasil, uma vez que apenas os herbicidas alachlor, sethoxydim e trifluralin possuem registro (Rodrigues \& Almeida, 1998). Desta forma, as opções são escassas, e as existentes basicamente controlam plantas daninhas de folhas estreitas. Poucos pesquisadore no país estudaram a viabilidade de herbicidas latifolicidas para a cultura do girassol (Rios \& Gimenez,1984; Dower Neto et al.; 1986; Vidal, 1990; Fleck \& Vidal, 1993; Vidal \& Fleck, 1993; Fleck \& Vidal, 1994; Brighenti et al., 2000a, 2000b, 2000c; Gazziero et al., 2001). Entretanto, ainda existe muitas dúvidas a respeito, principalmente na seletividade dos herbicidas ao girassol, o que enfatiza a carência de maiores estudos, e conseqüentemente, de novas alternativas para as diferentes regiões do Brasil.

O aclonifen é um herbicida do grupo químico dos difenil éteres e de uso consagrado em outros países, mas ainda as- sim, estão faltando trabalhos para viabilizar o seu uso isolado ou em misturas com outros herbicidas para as diferentes regiões do Brasil, tornando-o mais uma opção para a cultura do girassol.

O presente trabalho teve como objetivos avaliar a seletividade e a eficácia do aclonifen, isolado ou em mistura com quizalofop-p-ethyl, fomesafen e chlorimuron-ethyl, no controle em pós-emergência das plantas daninhas na cultura do girassol.

\section{MATERIAL E MÉTODOS}

O experimento foi conduzido na Fazenda Experimental da Universidade Estadual de Maringá, localizada no distrito de Iguatemi, pertencente ao Município de Maringá, Estado do Paraná. A semeadura convencional do girassol foi efetuada em 23/12/98, utilizando-se o híbrido Morgan 742 no espaçamento de $0,70 \mathrm{~m}$, com 4 a 5 sementes por metro. A adubação de semeadura consistiu em $300 \mathrm{~kg} / \mathrm{ha}$ do formulado 04-20-20 (NPK), e aos 28 dias após a semeadura aplicou-se 80 $\mathrm{kg} / \mathrm{ha}$ de uréia em cobertura. A colheita foi realizada em 26/03/ 99, de forma manual.

$\mathrm{O}$ solo do experimento apresentava $\mathrm{pH} 5,3 \mathrm{em}$ água, $2,92 \mathrm{cmol} \mathrm{de} \mathrm{H}^{+}+\mathrm{Al}^{+3} / \mathrm{dm}^{3}, 1,49 \mathrm{cmol}_{\mathrm{c}}$ de $\mathrm{Ca}^{+2}+\mathrm{Mg}^{+2} / \mathrm{dm}^{3}$, $0,06 \mathrm{cmolc}_{\mathrm{c}}^{\mathrm{c}} \mathrm{de} \mathrm{K}^{+} / \mathrm{dm}^{3}, 34 \mathrm{mg}$ de $\mathrm{P} / \mathrm{dm}^{3}, 5,32 \mathrm{~g} \mathrm{de} \mathrm{C} / \mathrm{dm}^{3}, 40 \%$ de areia grossa, $44 \%$ de areia fina, $1 \%$ de silte e $15 \%$ de argila.

O delineamento experimental utilizado foi blocos casualizados, com nove tratamentos e quatro repetições, sendo a área das parcelas de 4 x 5 metros $\left(20 \mathrm{~m}^{2}\right)$. Todos os tratamentos químicos (Tabela 1 ) foram aplicados em pós-emergência, em 21/01/1999, quando o girassol encontrava-se no estádio V6 a V8, utilizando um pulverizador costal de pressão constante a base de $\mathrm{CO}$, operando com pontas de jato plano XR $110-\mathrm{SF}-02$ a $2,0 \mathrm{~kg} f / \mathrm{cm}^{2}$, o que proporcionou um consumo de calda de $200 \mathrm{~L} / \mathrm{ha}$. Por ocasião da aplicação dos produtos o solo encontrava-se úmido, a temperatura do ar era de $31^{\circ} \mathrm{C}$, a umidade relativa do ar estava em $73 \%$, o céu sem nuvens e a velocidade do vento era de $2 \mathrm{~km} / \mathrm{h}$.

As plantas daninhas presentes na área experimental no momento da aplicação dos herbicidas foram constituídas por uma população de 75 plantas de Cenchrus echinatus (capim-carrapicho) $/ \mathrm{m}^{2}, 10$ plantas de Commelina benghalensis (trapoeraba) $/ \mathrm{m}^{2}, 11$ plantas de Portulaca oleracea (beldroega)/ $\mathrm{m}^{2}$ e 12 plantas de Emilia sonchifolia (falsa-serralha) $/ \mathrm{m}^{2}$. O estádio de desenvolvimento do $C$. echinatus era de 1 a 3 perfilhos, estando a maioria com 1 perfilho, a $P$ oleracea a $E$. sonchifolia com 2 a 4 folhas e a $C$. benghalensis com 2 a 3 folhas.

A percentagem de controle das espécies daninhas, o grau de fitotoxicidade (E.W.R.C., 1964) e a redução do porte da cultura (\%) foram avaliados aos 15, 30 e 45 dias após aplicação (DAA). O número de plantas de girassol/3 $\mathrm{m}$ e a dificuldade de colheita através de índices de 1 a 5 ( 1 = impossível de colher, 2 = colheita bastante problemática, $3=$ colheita 
Tabela 1. Tratamentos, formulação e doses utilizadas no experimento. Iguatemi, PR, 1998/99.

\begin{tabular}{|c|c|c|c|c|}
\hline \multirow[t]{2}{*}{ Tratamento } & \multicolumn{2}{|c|}{ Formulação } & \multicolumn{2}{|c|}{ Dose } \\
\hline & Tipo & Concentração & Ingrediente ativo & Produto comercial \\
\hline Testemunha sem capina & - & - & - & - \\
\hline Testemunha capinada & - & - & - & - \\
\hline Aclonifen $^{1}$ & SC & $600 \mathrm{~g} / \mathrm{L}$ & $780 \mathrm{~g} / \mathrm{ha}$ & $1,3 \mathrm{~L} / \mathrm{ha}$ \\
\hline Aclonifen & SC & $600 \mathrm{~g} / \mathrm{L}$ & $900 \mathrm{~g} / \mathrm{ha}$ & $1,5 \mathrm{~L} / \mathrm{ha}$ \\
\hline Quizalofop-p-ethyl ${ }^{2}$ & $\mathrm{CE}$ & $50 \mathrm{~g} / \mathrm{L}$ & $75 \mathrm{~g} / \mathrm{ha}$ & $1,5 \mathrm{~L} / \mathrm{ha}$ \\
\hline Aclonifen + quizalofop-p-ethyl & $\mathrm{SC}+\mathrm{CE}$ & $600 \mathrm{~g} / \mathrm{L}+50 \mathrm{~g} / \mathrm{L}$ & $900+75 \mathrm{~g} / \mathrm{ha}$ & $1,5 \mathrm{~L} / \mathrm{ha}+1,5 \mathrm{~L} / \mathrm{ha}$ \\
\hline Aclonifen + quizalofop-p-ethyl & $\mathrm{SC}+\mathrm{CE}$ & $600 \mathrm{~g} / \mathrm{L}+50 \mathrm{~g} / \mathrm{L}$ & $900+100 \mathrm{~g} / \mathrm{ha}$ & $1,51 / \mathrm{ha}+2,0 \mathrm{~L} / \mathrm{ha}$ \\
\hline Aclonifen + fomesafen $^{3}$ & $\mathrm{SC}+\mathrm{SAq}$ & $600 \mathrm{~g} / \mathrm{L}+250 \mathrm{~g} / \mathrm{L}$ & $900+25 \mathrm{~g} / \mathrm{ha}$ & $1,5 \mathrm{~L} / \mathrm{ha}+0,1 \mathrm{~L} / \mathrm{ha}$ \\
\hline Aclonifen + chlorimuron-ethyl ${ }^{4}$ & $\mathrm{SC}+\mathrm{GrDA}$ & $600 \mathrm{~g} / \mathrm{L}+250 \mathrm{~g} / \mathrm{kg}$ & $900+2,5 \mathrm{~g} / \mathrm{ha}$ & $1,5 \mathrm{~L} / \mathrm{ha}+0,01 \mathrm{~kg} / \mathrm{ha}$ \\
\hline
\end{tabular}

Obs.:- Nome comercial $={ }^{1}$ Prodigio $600 \mathrm{SC} ;{ }^{2} \mathrm{Targa} ;{ }^{3} \mathrm{Flex} ;{ }^{4} \mathrm{Classic}$

medianamente problemática, 4 = colheita levemente problemática e $5=$ sem problemas para colher) foram avaliados na précolheita da cultura. A produtividade do girassol foi obtida colhendo-se plantas de duas linhas com $4 \mathrm{~m}$ de comprimento na área útil das parcelas, com posterior transformação para $\mathrm{kg} / \mathrm{ha}$.

Os dados obtidos foram submetidos a análise de variância e as médias comparadas pelo teste de Tukey ao nível de $5 \%$ de probabilidade.

\section{RESULTADOS E DISCUSSÃO}

Nos tratamentos com quizalofop-p-ethyl, isolado ou em mistura com aclonifen, os índices de controle de $C$. echinatus foram excelentes (100\%) aos 15 DAA (Tabela 2). E estes ainda mantiveram controle acima de $97 \%$ aos 30 e 45 DAA, não diferindo significativamente da testemunha capinada. Estes resultados demonstraram que o quizalofop-p-ethyl foi o principal responsável pelo controle da planta daninha, sendo que a mistura com aclonifen não demonstrou efeito antagônico que fosse prejudicial à eficácia do produto. Para aclonifen isolado ou em mistura com fomesafen e chlorimuronethyl, os níveis de controle de $C$. echinatus não foram satisfatórios em nenhuma das avaliações. Entretanto, o aclonifen apresenta alguma ação sobre esta gramínea, sendo que o controle com $900 \mathrm{~g} / \mathrm{ha}$ foi superior à dose de $780 \mathrm{~g} / \mathrm{ha} \mathrm{em}$ todas avaliações, apesar de terem atingido níveis insuficientes de eficiência ( $\leq 54 \%$ ). Para a $C$. benghalensis (Tabela 2), o controle foi nulo para o quizalofop-p-ethyl isolado (75 g/ha) em todas as avaliações. Os demais tratamentos controlaram a C. benghalensis com elevada eficácia, não diferindo da testemunha capinada. Os resultados demonstraram grande susceptibilidade da espécie $C$. benghalensis em relação ao herbicida aclonifen, uma vez que o mesmo não necessitou do fomesafen ou chlorimuron-ethyl, em mistura, para obter controle de $100 \%$.

A espécie $P$. oleracea apresentou os mesmos resultados da C. benghalensis, ou seja, o quizalofop-p-ethyl isolado obteve controle nulo e os demais tratamentos químicos controlaram de forma excelente a infestante, não diferindo da testemunha capinada aos 45 DAA (Tabela 3). O aclonifen isolado, nas doses de 780 e $900 \mathrm{~g} / \mathrm{ha}$, proporcionaram controle de $100 \%$ da P. oleracea. Para E. sonchifolia, apenas a mistura de aclonifen + chlorimuron-ethyl $(900+2,5 \mathrm{~g} / \mathrm{ha})$ atingiu $100 \%$ de controle aos $45 \mathrm{DAA}$. Todos os demais tratamentos foram de baixa eficácia para esta espécie. Os resultados demonstram que o aclonifen isolado (780 e $900 \mathrm{~g} / \mathrm{ha}$ ) apresenta deficiência no controle de $E$. sonchifolia, mas em mistura com chlorimuronethyl $(900+2,5 \mathrm{~g} / \mathrm{ha})$ pode vir a ser uma boa alternativa para ampliar o espectro de controle de folhas largas.

Apenas o quizalofop-p-ethyl, isolado ou em mistura com aclonifen, proporcionou sintomas visíveis de injúrias pela escala E.W.R.C., as quais inicialmente consistiram de encarquilhamento dos ponteiros das plantas aos 15 DAA (Tabela 4). Aos $30 \mathrm{DAA}$ estes sintomas já não eram mais visíveis. Com relação ao desenvolvimento da cultura, todos os tratamentos químicos reduziram visualmente o porte do girassol aos 15 DAA. Esta redução foi menos expressiva para aclonifen isolado, apresentando-se em torno de 10 a $15 \%$; para aclonifen + quizalofop-p-ethyl a redução foi de 30 a $35 \%$; de $40 \%$ para aclonifen + fomesafen e redução mais severa para aclonifen + chlorimuron-ethyl $(900+2,5 \mathrm{~g} / \mathrm{ha})$, a qual atingiu $60 \%$. A partir dos 30 DAA a cultura encontrava-se em pleno processo de recuperação, sendo que aos 45 DAA já não era possível observar visualmente maiores reduções de porte nas duas doses de aclonifen isolado. Aos 45 DAA, para o quizalofop-p-ethyl e as misturas de aclonifen + quizalofop-p-ethyl a cultura ainda apresentava uma redução de porte em torno de 5\%; para aclonifen + fomesafen de $10 \%$ e de $15 \%$ para aclonifen + chlorimuron-ethyl.

Na pré-colheita não era visível a redução do porte da cultura nos tratamentos químicos, quando comparados à testemunha capinada. Aos 30 DAA, observou-se que a abertura do capítulo das plantas nos tratamentos aclonifen + quizalofop-p-ethyl, aclonifen + fomesafen e aclonifen + chlorimuron-ethyl estava atrasada em relação aos demais tratamentos. Aos 45 DAA, todos os tratamentos apresentaram 
Cleber Daniel de G. Maciel et al.

Tabela 2. Porcentagens de controle de Cenchrus echinatus e Commelina benghalensis aos 15, 30 e 45 DAA, na cultura do girassol. Iguatemi, PR, 1998/99.

\begin{tabular}{|c|c|c|c|c|c|c|c|}
\hline \multirow[t]{2}{*}{ Tratamento } & \multirow{2}{*}{$\begin{array}{l}\text { Dose } \\
\text { (g/ha) }\end{array}$} & \multicolumn{3}{|c|}{ Cenchrus echinatus } & \multicolumn{3}{|c|}{ Commelina benghalensis } \\
\hline & & 15 DAA & 30 DAA & 45 DAA & 15 DAA & 30 DAA & 45 DAA \\
\hline Testemunha sem capina & - & $0 \mathrm{~d}$ & $0 \mathrm{~d}$ & $0 \mathrm{~d}$ & 0 & $0 \mathrm{~b}$ & 0 \\
\hline Testemunha capinada & - & $100 \mathrm{a}$ & $100 \mathrm{a}$ & $100 \mathrm{a}$ & 100 & $100 \mathrm{a}$ & 100 \\
\hline Aclonifen & 780 & $50 \mathrm{c}$ & $26 \mathrm{c}$ & $39 \mathrm{c}$ & 100 & $100 \mathrm{a}$ & 100 \\
\hline Aclonifen & 900 & $71 \mathrm{~b}$ & $52 \mathrm{~b}$ & $54 \mathrm{~b}$ & 100 & $100 \mathrm{a}$ & 100 \\
\hline Quizalofop-p-ethyl & 75 & $100,00 \mathrm{a}$ & 98 a & $98 \mathrm{a}$ & 0 & $0 \mathrm{~b}$ & 0 \\
\hline Aclonifen + quizalofop-p-ethyl & $900+75$ & $100,00 \mathrm{a}$ & 99 a & 99 a & 100 & $100 \mathrm{a}$ & 100 \\
\hline Aclonifen + quizalofop-p-ethyl & $900+100$ & $100,00 \mathrm{a}$ & 98 a & 98 a & 100 & $100 \mathrm{a}$ & 100 \\
\hline Aclonifen + fomesafen & $900+25$ & $60 \mathrm{bc}$ & $46 \mathrm{~b}$ & $51 \mathrm{~b}$ & 100 & $100 \mathrm{a}$ & 100 \\
\hline Aclonifen + chlorimuron-ethyl & $900+2,5$ & $56 \mathrm{bc}$ & $40 \mathrm{bc}$ & $45 \mathrm{bc}$ & 100 & $100 \mathrm{a}$ & 100 \\
\hline $\mathrm{F}$ & - & $116,81^{*}$ & $94,9 *$ & $231,3^{*}$ & - & $279801,0^{*}$ & - \\
\hline C.V.(\%) & - & 8,9 & 12,4 & 7,2 & - & 0,2 & - \\
\hline D.M.S. $(5 \%)$ & - & 15,1 & 18,6 & 11,3 & - & 0,4 & - \\
\hline
\end{tabular}

OBS: - DAA= Dias após aplicação

- Médias, na mesma coluna, seguidas da mesma letra não diferem significativamente entre si.

$-*(\mathrm{p}<0,05)$

Tabela 3. Porcentagens de controle de Portulaca oleracea e Emilia sonchifolia aos 15, 30 e 45 DAA, na cultura do girassol. Iguatemi, PR, 1998/99.

\begin{tabular}{lccccccc}
\hline \multirow{2}{*}{ Tratamento } & Doses & \multicolumn{3}{c}{ Portulaca oleracea } & \multicolumn{3}{c}{ Emilia sonchifolia } \\
\cline { 3 - 8 } & $(\mathrm{g} / \mathrm{ha})$ & 15 DAA & 30 DAA & 45 DAA & 15 DAA & 30 DAA & 45 DAA \\
\hline Testemunha sem capina & - & 0 & $0 \mathrm{c}$ & $0 \mathrm{~b}$ & $0 \mathrm{c}$ & $0 \mathrm{c}$ & $0 \mathrm{c}$ \\
Testemunha capinada & - & 100 & $100 \mathrm{a}$ & $100 \mathrm{a}$ & $100 \mathrm{a}$ & $100 \mathrm{a}$ & $100 \mathrm{a}$ \\
Aclonifen & 780 & 100 & $99 \mathrm{a}$ & $100 \mathrm{a}$ & $17 \mathrm{bc}$ & $23 \mathrm{~b}$ & $12 \mathrm{bc}$ \\
Aclonifen & 900 & 100 & $100 \mathrm{a}$ & $100 \mathrm{a}$ & $14 \mathrm{bc}$ & $21 \mathrm{~b}$ & $12 \mathrm{bc}$ \\
Quizalofop-p-ethyl & 75 & 0 & $00 \mathrm{c}$ & $0 \mathrm{~b}$ & $0 \mathrm{c}$ & $0 \mathrm{c}$ & $0 \mathrm{c}$ \\
Aclonifen + quizalofop-p-ethyl & $900+75$ & 100 & $100 \mathrm{a}$ & $100 \mathrm{a}$ & $29 \mathrm{~b}$ & $27 \mathrm{~b}$ & $12 \mathrm{bc}$ \\
Aclonifen + quizalofop-p-ethyl & $900+100$ & 100 & $94 \mathrm{~b}$ & $99 \mathrm{a}$ & $22 \mathrm{~b}$ & $17 \mathrm{bc}$ & $10 \mathrm{bc}$ \\
Aclonifen + fomesafen & $900+25$ & 100 & $97 \mathrm{~b}$ & $100 \mathrm{a}$ & $29 \mathrm{~b}$ & $24 \mathrm{~b}$ & $21 \mathrm{~b}$ \\
Aclonifen + chlorimuron-ethyl & $900+2,5$ & 100 & $100 \mathrm{a}$ & $100 \mathrm{a}$ & $100 \mathrm{a}$ & $97 \mathrm{a}$ & $100 \mathrm{a}$ \\
\hline F & - & - & $1555,50^{*}$ & $31045,44^{*}$ & $69,46^{*}$ & $80,03^{*}$ & $231,02^{*}$ \\
C.V.(\%) & - & - & 2,9 & 0,6 & 26,7 & 24,4 & 17,8 \\
D.M.S. (5\%) & - & - & 5,3 & 1,2 & 22,2 & 20,3 & 12,8 \\
\hline
\end{tabular}

OBS: - DAA= Dias após aplicação

- Médias, na mesma coluna, seguidas da mesma letra não diferem significativamente entre si.

$-*(\mathrm{p}<0,05)$

abertura uniforme do capítulo das plantas, semelhante à testemunha capinada, sendo que nas misturas de aclonifen + quizalofop-p-ethyl e aclonifen + chlorimuron-ethyl, ocorreu o aparecimento de capítulos nas gemas laterais em algumas plantas, fato este não observados nos demais tratamentos e na testemunha capinada. De maneira geral, pode-se verificar que $o$ aclonifen isolado provocou os menores sintomas iniciais de fitotoxicidade, e que nenhum tratamento químico ou mesmo a condição de mato interferência da testemunha sem capina afetou o estande da cultura.

Com relação à produção, apenas os tratamentos onde o controle, principalmente de $C$. echinatus apresentou-se eficiente a produção não foi afetada, igualando-se à testemunha capinada. É importante ressaltar que o C. echinatus dominou o experimento com o passar do tempo, suprimindo o desenvolvimento das demais plantas daninhas presentes na área experimental, e portanto, tornou-se o maior responsável pela interferência na produção do girassol. Desta forma, os tratamentos com aclonifen isolado e as misturas aclonifen + fomesafen e aclonifen + chlorimuron-ethyl, por não terem controlado eficientemente o $C$. echinatus tiveram sua produção afetada. Com relação à dificuldade de colheita, houve a mesma tendência observada para o parâmetro de produção, onde as maiores dificuldades encontradas foram constatadas para os tratamentos que não controlaram eficazmente o C. echinatus. Para os tratamentos com quizalofop-p-ethyl, que proporcionou excelente controle de $C$. echinatus, a colheita procedeu- 
Tabela 4. Toxicidade (E.W.R.C.) e redução do porte da cultura (\%), número de plantas/3 m lineares na pré-colheita e produtividade da cultura do girassol, submetida aos diversos tratamentos. Iguatemi, PR, 1998/99.

\begin{tabular}{|c|c|c|c|c|c|c|c|}
\hline \multirow[t]{2}{*}{ Tratamento } & \multirow{2}{*}{$\begin{array}{l}\text { Dose } \\
\text { (g/ha) }\end{array}$} & \multicolumn{3}{|c|}{$\begin{array}{c}\text { Fitotoxicidade } \\
\text { Reducãode }\end{array}$} & \multirow[b]{2}{*}{$\begin{array}{l}\text { Plantas / 3m } \\
\text { Pré-colheita }\end{array}$} & \multirow[b]{2}{*}{$\begin{array}{l}\text { Produtividade } \\
\text { (kg/ha) }\end{array}$} & \multirow[b]{2}{*}{$\begin{array}{l}\text { Dificuldade de } \\
\text { Colheita }\end{array}$} \\
\hline & & $\frac{\text { Red }}{15 \text { DAA }}$ & $\frac{\text { cão de altur }}{30 \text { DAA }}$ & $\frac{(\%)}{45 \text { DAA }}$ & & & \\
\hline Testemunha sem capina & - & $1(0 \%)$ & 1 & $1(0 \%)$ & $7,75 \mathrm{a}$ & $897,14 \mathrm{c}$ & 2,00 \\
\hline Testemunha capinada & - & $1(0 \%)$ & 1 & $1(0 \%)$ & $10,25 \mathrm{a}$ & 2082,97 a & 5,00 \\
\hline Aclonifen & 780 & $1(10 \%)$ & 1 & $1(0 \%)$ & $7,75 \mathrm{a}$ & $1039,29 \mathrm{bc}$ & 2,25 \\
\hline Aclonifen & 900 & $1(15 \%)$ & 1 & $1(0 \%)$ & $7,25 \mathrm{a}$ & $1066,43 \mathrm{bc}$ & 2,25 \\
\hline Quizalofop-p-ethyl & 75 & $3(30 \%)$ & 1 & $1(5 \%)$ & $7,75 \mathrm{a}$ & $1719,28 \mathrm{ab}$ & 4,25 \\
\hline Aclonifen + quizalofop-p-ethyl & $900+75$ & $3(35 \%)$ & 1 & $1(5 \%)$ & $8,50 \mathrm{a}$ & $1791,79 \mathrm{ab}$ & 4,25 \\
\hline Aclonifen + quizalofop-p-ethyl & $900+100$ & $3(35 \%)$ & 1 & $1(5 \%)$ & $10,50 \mathrm{a}$ & 2024,99 a & 4,50 \\
\hline Aćlonifen + fomesafen & $900+25$ & $1(40 \%)$ & 1 & $1(10 \%)$ & $8,00 \mathrm{a}$ & $891,43 c$ & 2,25 \\
\hline Aclonifen + chlorimuron-ethyl & $900+2,5$ & $1(60 \%)$ & 1 & $1(15 \%)$ & $7,50 \mathrm{a}$ & $757,14 \mathrm{c}$ & 2,25 \\
\hline$\overline{F-}$ & - & - & - & $1,63^{\mathrm{NS}}$ & $11,11^{*}$ & - & \\
\hline C.V.(\%) & - & - & - & - & 22,4 & 23,4 & - \\
\hline D.M.S. $(5 \%)$ & - & - & - & - & 4,50 & 767,83 & - \\
\hline
\end{tabular}

OBS: - DAA= Dias após aplicação

- Médias, na mesma coluna, seguidas da mesma letra não diferem significativamente entre si.

$-*(\mathrm{p}<0,05) ;-{ }^{\mathrm{NS}}=$ Não significativo.

- A identificação da redução de altura das plantas tomou como base a testemunha capinada.

- Dificuldade de colheita: 1 = imposșível de colher......5 = sem problemas para colher.

se praticamente sem problemas. A interferência das plantas daninhas reduziu em média $50 \%$ a produção do girassol, resultados estes semelhantes aos relatados por Constantin (1994), Fleck (1989) e Robinson (1966).

\section{CONCLUSÕES}

O aclonifen + quizalofop-p-ethyl $(900+75$ e $900+100 \mathrm{~g} /$ ha) e o quizalofop-p-ethyl isolado ( $75 \mathrm{~g} / \mathrm{ha}$ ) foram excelentes no controle de $C$. echinatus.

$\mathrm{O}$ aclonifen isolado (780 e $900 \mathrm{~g} / \mathrm{ha}$ ) e suas misturas com fomesafen e chlorimuron-ethyl $(900+25$ e $900+2,5 \mathrm{~g} / \mathrm{ha})$ não foram eficientes no controle de $C$. echinatus, mas apresentaram excelente controle de $C$. benghalensis e $P$. oleracea.

Apenas a mistura de aclonifen + chlorimuron-ethyl $(900$ $+2,5 \mathrm{~g} / \mathrm{ha}$ ) obteve controle eficiente de E. sonchifolia.

Os tratamentos químicos não prejudicaram o desenvolvimento final e o estande da cultura; a produtividade do girassol foi positivamente influenciada pelo controle de $C$. echinatus.

A interferência das plantas daninhas, constituídas principalmente de $C$. echinatus, dificultou a colheita e reduziu a produção do girassol em $50 \%$.

\section{LITERATURA CITADA}

\author{
BALLA, A.; CASTIGLIONI, V.B.R.; CASTRO, C. Colheita de \\ girassol. Londrina: EMBRAPA-CNPSo, 1995. 25p. \\ (EMBRAPA-CNPSo, Documentos, 92).
}

BRIGHENTI, A.M.; GAZZIERO, D.L.P.; OLIVEIRA, M.F.; VOLL, E.; PEREIRA, J.E. Controle de plantas daninhas na cultura do girassol em solo de textura argilosa. Revista Bras. Herb., v.1, n.1, p.85-88, 2000a.

BRIGHENTI, A.M.; FORNAROLLI, D.A.; OLIVEIRA JÚNIOR, R.S.; GAZZIERO, D.L.P.; PINTO, R. Seletividade de herbicidas aplicados em condições de pré-emergência da cultura do girassol. Revista Bras. Herb., v.1, n.3, p.243$247,2000 b$.

BRIGHENTI, A.M.; OLIVEIRA, M.F.; GAZZIERO, D.L.P.; PEREIRA, J.E.; VOLL, E. Sunflower tolerance to preemergence broadleaf weed herbicides. In: INTERNATIONAL SUNFLOWER CONFERENCE, 15. Toulouse, France, 2000. Actes Proceedings. Tomo I. p.73$77,2000 \mathrm{c}$.

CASTRO, C.; CASTIGLIONI, V.B.R.; BALLA, A.; LEITE, R.M.V.B.C.; KARAM, D.; MELLO, H.C.; GUEDES, L.C.A.; FARIAS, J.R.B. A cultura do girassol. Londrina: EMBRAPA-CNPSo, 1996.36p. (EMBRAPA-CNPSo, Circular Técnica, 13).

CONSTANTIN, J. Instalação da cultura do girassol. In: BRINHOLI, D. Cultura do girassol (Helianthus annuus L.). Botucatu: UNESP, 1994. p.101-127.

CHUBB, W.O. Weed competition in suwflowers. Agron. Conf. Tech., Manitoba, p.119-132, 1975.

DOWER NETO, J.B.; COPPO, O.J.; JORGE; M.S.; MEZZON, A.; NEGRÃO, E.A.; REIS, L.C.; ROSSI, W.B.; SILVA, N.R. 
Estudo comparativo de herbicidas pós-emergentes para controle de plantas daninhas e fitotoxicidade na cultura de girassol (Helianthus annuus L.). In: CONGRESSO BRASILEIRO DE HERBICIDAS E PLANTAS DANINHAS, 16. Campo Grande, 1986, Resumos.... Campo Grande: SBHED, 1986. p.97.

EUROPEAN WEED RESEARCH COUNCIL-EWRC. Methods in weeds research. Weed Research, Oxford, v.4, p.88, 1964.

FLECK, N.G. Interferência de plantas daninhas na cultura do girassol. Competição no tempo. Pesq. Agropec. Bras., Brasília, v.24, n.9, p.1139-1147, 1989.

FLECK, N.G.; VIDAL, R.A. Injúria potencial de solo ao girassol. II. Chlorimuron-ethyl. Planta Daninha, Brasília, v.11, n. $1 / 2$, p.44-48, 1993.

FLECK, N.G.; VIDAL, R.A. Injúria potencial de herbicidas de solo ao girassol. III - Imazaquin e imazethapyr. Planta Daninha, Brasília, v.12, n.1, p.39-43, 1994.

GAZZIERO, D.L.P.; BRIGHENTI, A.M.; CASTRO, C.; PRETE, C.E.C.; VOLL, E. Comportamento do girassol quando cultivado em área tratadas com o herbicida 2,4-D. Planta Daninha, v.19, n.1, p.127-133, 2001.

MONTES de OCA, C.Y.; PEARSOM, F. Control de malezas anuales en el cultivo de girasol. AACREA - XII Congresso Zona Mar Y Sierras, 1987.

PIRES, J.C. Cultura do girassol (Helianthus annuus L.): Introdução, botânica e melhoramento. In: BRINHOLI, D. Cul- tura do girassol (Helianthus annuus L.). Botucatu: UNESP, 1994.p.1-25

.RIOS, A.; GIMENEZ, A. Control de malezas en girassol (Helianthus annuus L.). In: CONGRESSO BRASILEIRO DE HERBICIDAS E ERVAS MEDICINAIS, 15 e CONGRESSO DE ASOCIACIONLATINOAMERICANA DE MALEZAS, 7. Belo Horizonte, 1984. Resumos....Belo Horizonte: SBHED, 1984. p.109.

ROBINSON, R.G. Sunflower, soybean and grain sorghum-corn rotations versus monoculture. Agronomy Journal, v.58, p.475-477, 1966

RODRIGUES, B.N.; ALMEIDA, F.S. Guia de herbicidas. 4 ed. Londrina: Ed. dos Autores, 1998. 648p.

TRASMONTE, D.; DUARTE, G. Control de malezas en girasol. AACREA - V Congreso Zona Oeste Arenoso, 1991.

UNGARO, M.R.G. Instruções para a cultura do girassol. Campinas: IAC, 1978. 15p. (Boletim, 212)

VIDAL, R.A. Avaliações de herbicidas de pós-emergência para controle de plantas daninhas espontâneas de girassol. In: REUNIÃO NACIONAL DE PESQUISA DE GIRASSOL, 8. Cruz Alta, 1990. Resumos....Fundação Centro de Experimentação e Pesquisa Fecotrigo, 1990. p.33-34.

VIDAL, R.A.; FLECK, N.G. Injúria potencial de herbicidas de solo ao girassol. I - Clomazone. Planta Daninha, Brasília, v.11, n.1/2, p.36-43, 1993. 